\title{
Optimizing the energy efficiency of higher education institutions
}

\author{
Sergey Sergeev ${ }^{1, *}$, Sergey Barykin ${ }^{1}$, Olga Kalinina $^{1}$, Elena Naumova $^{2}$, Natalia \\ Dedyukhina $^{3}$, and Tatiana Dmitrieva ${ }^{4}$ \\ ${ }^{1}$ Peter the Great St. Petersburg Polytechnic University, Graduate School of Service and Trade, \\ 195251, St. Petersburg, Russia \\ ${ }^{2}$ Saint Petersburg State Maritime Technical University, Engineering and Economics Faculty, 190121, \\ St. Petersburg, Russia \\ ${ }^{3}$ Emperor Alexander I St. Petersburg State Transport University, Department of Accounting and \\ Audit, 190031, St. Petersburg, Russia \\ ${ }^{4}$ Peter the Great St. Petersburg Polytechnic University, Graduate School of Management and \\ Business, 195251, St. Petersburg, Russia
}

\begin{abstract}
The article presents the results of research conducted at the St. Petersburg Polytechnic University within the framework of the innovative business incubator and aimed at forming a cross-system approach to energy consumption management. The authors consider the features being typical for most educational institutions, regardless of the profile and specifics. Constant components of energy consumption are taken into account, such as outdoor lighting, scientific equipment, security systems, fire extinguishing systems, equipment of the dining room and buffet, and engineering services. The basis is a mathematical model that allows not only to describe the processes, but also to apply the methods of optimization theory and develop software for effective management of engineering equipment. The authors have used a search method based on the analysis of nonlinear functions. With the purpose of the research, an application with an extension for finding solutions to optimization problems was used in the free software package with the Linux kernel.
\end{abstract}

\section{Introduction}

Familiarization with the experience of universities in advanced countries in improving the efficiency of energy use allowed us to draw a number of conclusions, primarily about the role played by a systematic approach to this problem. The industry that pollutes nature spends the most energy [1]. Energy management is essential in both financial and environmental aspects [2]. Such important questions as energy shortage and increase in GHG emissions, have raised many concerns worldwide about current trends in energy consumption [3]. The green building technologies in China are studied in [4]. China's energy saving in different fields are investigated by [5]. The building energy savings business model needs integration with other housing association services in Finland [6].

\footnotetext{
*Corresponding author: sergeev2@inbox.ru
} 
Some researchers focuse on the viability of a business model based on energy efficiency contracts [7], energy efficiency services [8], energy-efficient system resulting in environmental benefits for the project stakeholders and the entire society [9]. Energy savings opportunities may be explored through the deployment of solar hot water/steam generation systems to reduce carbon emissions from the plant [10].

The environmental innovations are discussed from the green management point of view [11]. Some issues regarding the impacts on reducing waste, pollution and emission of greenhouse gases are studied in [12]. The ways to provide the energy efficiency to customers are considered from the energy services business model point of view [13]. Energy service companies are expected to play an important role in promoting energy efficiency [14].

Energy efficiency program in the public sector of Russia is based on its enormous potential for energy conservation [15]. In recent years, the attention to the issues of economy in the walls of educational institutions has increased dramatically including some issues regarding the innovations [16] and digitalization [17]. This is due not only to the continuous increase in prices for used energy resources (ER) and the desire to balance the budget, but also to such important factors as taking into account the prospect of depletion of natural resources, the impact of environmental requirements and the long-term interests of future generations.

In a number of research centers [18] a lot of work is being done to find reserves for saving the main types of heat, electricity, fuel consumption and other resources. At the same time, it is necessary to act in conditions of strict restrictions [19] to ensure comfortable conditions for conducting educational and research processes, compliance with the regulations on labor protection and standards of illumination, humidity and other key factors. Another limiting condition is the budget of the higher education institution, which determines the limit of funds for solving this problem. In addition, the adopted program clearly defines the directions and levels of reducing the absolute figures of energy consumption. In particular, we note such items as:

- organizational measures for energy saving and improvement of energy efficiency

energy efficiency of these organizations

- conducting energy surveys of buildings, structures, structures

- development of feasibility studies for implementation

energy-saving technologies

- technical and technological measures for energy saving and

improving the energy efficiency of these organizations

- control of thermal energy consumption by buildings

- wide automation

- energy efficiency of lighting systems

- replacement of inefficient, obsolete systems and equipment.

The fact is that although similar installations operate in many developed countries, a common approach to the integrated formation of an optimal energy consumption strategy has not yet been identified [20]. There is also no generally accepted methodology for forecasting such activities, which is necessary when drawing up plans and budgets.

This is due to the fact that the issue was primarily dealt with by energy practitioners, while here it is necessary to use more complex systems [21], not only computer modeling, but also to involve the complex mathematical apparatus of the theory of optimal systems [22]. 


\section{Problem statement}

Within the framework of the business incubator of the St. Petersburg Polytechnic University [23], research work was carried out aimed at finding approaches to solving the problem of energy saving in the university, as well as developing a scientifically based methodology for [24] planning the costs of energy support for the activities of the educational institution.

When forming the task statement, the following features were taken into account, which are typical for most educational institutions, regardless of the profile and specifics:

- the schedule of placement of staff and students in advance for half a year is defined, which is fixed by the schedule of classes and curricula.

- famous quantitative composition of persons in a specific classrooms and the main characteristics of the premises (volume, power, thermal and electric appliances, heat transfer to the environment, etc.).

- based on the forecast data, the known dependence of the ambient temperature, photoperiod, and other parameters meteorological conditions.

In addition, constant components of energy consumption are taken into account, such as outdoor lighting, scientific equipment, security systems, fire extinguishing systems, equipment of the dining room and buffet, and engineering services. The analysis assumes the presence of devices for regulating the supply of thermal energy and devices for maintaining the temperature in the premises, as well as presence sensors for controlling lighting devices.

The authors find interesting the fact that the schedule generation program offers a large number of options to choose from [25], which makes it possible to optimize.

\section{Mathematical model}

The mathematical model includes a matrix describing the nature of the temperature change in the rooms in the interval between the switching on of the thermostats. For the elements of this matrix, the heat transfer relations are used, and the incremental characteristic has an approximation of the form:

$$
t_{a}=K_{h}+Q^{*} \ln \left(\gamma^{*} \Delta T\right)
$$

where $t_{a}$ - indoor temperature, $K_{h}, Q, \gamma-$ coefficients that reflect the properties of this object, $\Delta T$ - time after switching on the heating devices;

the temperature decrement is accordingly described as

$$
t_{a}=K_{s}+D^{*} \exp (\lambda * \Delta T)
$$

where $t_{a}, \Delta T$ - have the same meaning, a $K_{s}, D, \lambda$ - similar coefficients that reflect the properties of this object after the heating devices are turned off.

The determination of the listed parameters $K_{h}, Q, \gamma, K_{s}, D, \lambda$ can theoretically [26] be performed from the Fourier equation [27]:

$\frac{\partial \mathrm{G}}{\partial \mathrm{t}}=W_{n} \frac{\partial^{2} G}{\partial t^{2}}+\Omega_{m}(x, t) \quad$ where $W_{n}, \Omega_{n}-$ relevant characteristics $n$-th room used in the educational process, research activities [28] for administrative and engineering purposes.

However, since the optimization problem requires relative values rather than absolute values [29], the problem is simplified and the desired characteristics can be obtained by 
examining the premises using time slices of temperatures determined by digital sensors, followed by processing on a computer [30].

Figure 1,2 shows the results of computer simulation of the process of changing the temperature in the room.

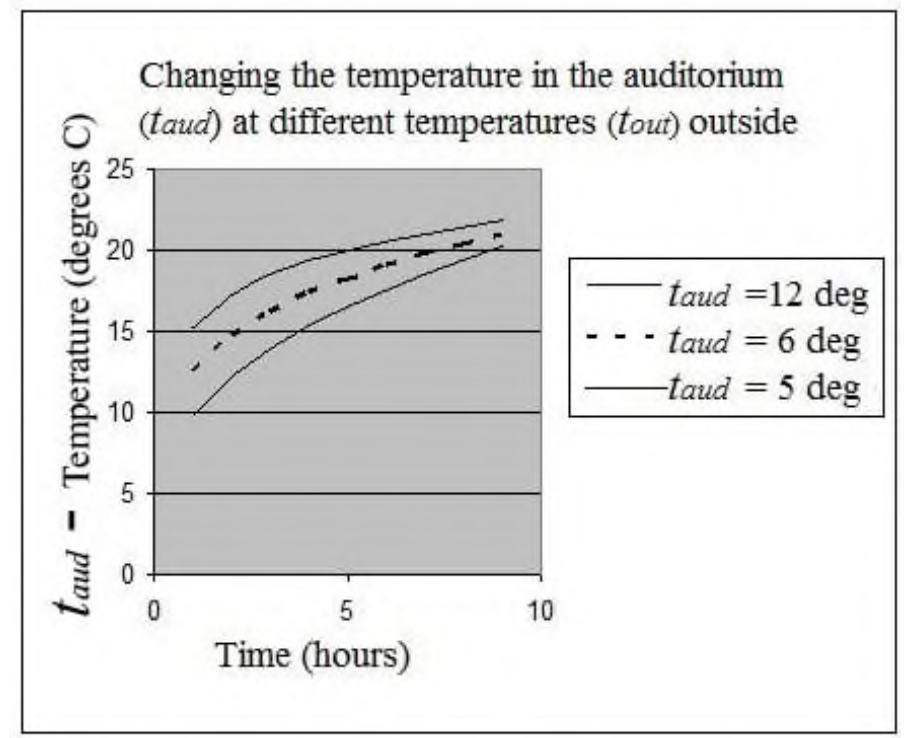

Fig. 1. The nature of the temperature increase in the room after turning on the heating at full capacity.

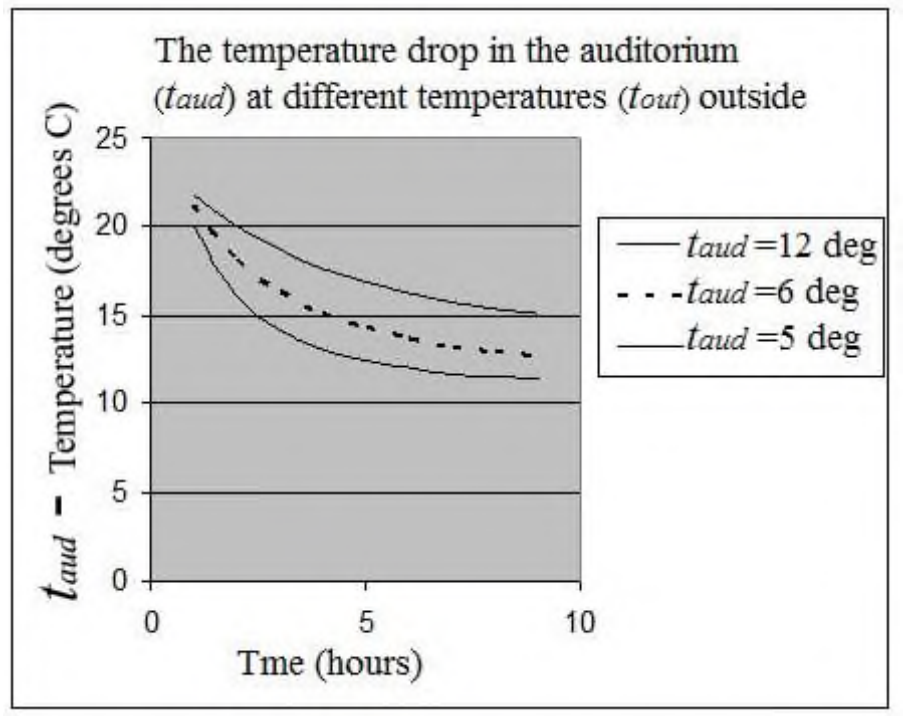

Fig. 2. The nature of the decrease in temperature in the room after turning off the heating.

The next step was to determine the coefficients [31] of the seasonal graph of ambient temperatures, which [32] was decomposed into a Fourier series:

$$
t^{*}(x)=A_{0}+\sum_{z=1}^{+\infty} A_{z} \cos \left(2 \pi \frac{z}{F} x+\theta_{z}\right)
$$


where the period is a calendar year, $x$ - where the period is a calendar year, the number of the harmonic decomposition, $F-$ period, $A_{z}-$ the corresponding coefficients of, $\theta_{z}-$ initial phase. As a result, we have [33] a periodic function that gives the value of the average temperature during the year, which allows us to apply a similar (3) decomposition, but with a period of 24 hours, to obtain a graph of the daily change in ambient temperature.

\section{Results}

Now, after applying optimization methods and selecting the minimum functional energy consumption as the criterion, it is necessary to choose the [34] distribution of training sessions from the options offered by the scheduling program at the first stage.

We used a search method based on the analysis of nonlinear functions. With the purpose of the research, an application with an extension for finding solutions to optimization problems was used in the free software package with the Linux kernel installed at the St. Petersburg State Polytechnic University.

The criterion included data from the matrix of room characteristics, temperature dependencies, and schedule.

At the first stage, the problem of heat supply is solved. The search for the optimal solution that provides the minimum of the objective function is carried out by calling the main procedure for finding solutions. The choice of the solver mechanism was carried out as a nonlinear one. The installed Solver for Nonlinear Programming program offers a choice of either the DEPS Evolutionary Algorithm or the SCO Evolutionary Algorithm. DEPS (Differential Evolution \& Particle Swarm Optimization ) consists of two independent algorithms and represents each solution vector as coordinates in the argument dimension space, simultaneously selecting one of the two strategies in each iteration and applying it to the current solution vector. SCO (Social Cognitive Optimization) simulates the behavior of learning and sharing information.

At each iteration, the system searches for a solution, comparing it with the data already received. Since there are no restrictive conditions defined separately by dependent inequalities in the problem described here, we can choose any mechanism. After the program is finished and the result is obtained, the desired parameters are stored in the computer's memory and can be used for automatic control of the control devices.

\section{Conclusions}

It should be noted that in representations (1) and (2), the simplest relay control mode was assumed [35]. This makes practical sense, since it significantly reduces both the cost of the equipment itself and its installation, and also simplifies the management interface [36]. Further, from the results obtained, another optimization reserve is visible [37].

The fact is that the seasonality equations of type (3) are only needed for advanced planning when making a budget, and during the current work $t(x)$, the values are simply replaced by the readings of digital sensors of the current temperature.

The calculations have shown that even only on heat carriers it is possible to save 14,200 Gcal per year, which in value terms leaves a value of about 3 million rubles, which is comparable to the planned figures of the energy efficiency program. The authors see further development of this system in the extension of the results to the consumed electricity, more accurate accounting of the parameters in equations (1), (2) by taking into account the topology of buildings and structures, clear taxation, and structural reorganization. Even the results of determining the above-mentioned coefficients of equivalent heat losses show the 
available reserves, which gives direction for ongoing engineering work, leading to savings in funds and resources.

\section{References}

1. T. Bhattacharya and R. Kapoor, Renewable and Sustainable Energy Reviews 16, 1311 (2012)

2. A. Mokhtar and M. Nasooti, Energy Strategy Reviews 28, 100458 (2020)

3. U. Berardi, Procedia Engineering 118, 128 (2015)

4. Y. Zhang, J. Kang, and H. Jin, Energies 11 (2018)

5. Y. F. Wang, K. P. Li, X. M. Xu, and Y. R. Zhang, Renewable and Sustainable Energy Reviews 29, 641 (2014)

6. N. Suhonen and L. Okkonen, Energy Policy 61, 783 (2013)

7. S. Pätäri and K. Sinkkonen, Journal of Cleaner Production 66, 264 (2014)

8. B. Duplessis, J. Ô. Adnot, M. Dupont, and F. Racapé, Energy Policy 45, 268 (2012)

9. X. Zhang, Z. Wu, Y. Feng, and P. Xu, Journal of Cleaner Production 109, 166 (2015)

10. A. K. K. Pandey and R. Prakash, Open Journal of Energy Efficiency 07, 89 (2018)

11. J. Sarkis, Journal of Cleaner Production 11, 397 (2003)

12. Y. Kayikci, Procedia Manufacturing 21, 782 (2018)

13. E. Vine, H. Nakagami, and C. Murakoshi, 24, 479 (2000)

14. E. Vine, Energy Policy 33, 691 (2005)

15. V. Roshchanka and M. Evans, Journal of Cleaner Production 112, 3905 (2016)

16. S. Barykin and A. Kobicheva, MATEC Web of Conferences (2018)

17. S. Barykin, A. Borovkov, O. Rozhdestvenskiy, A. Tarshin, and V. Yadykin, IOP Conference Series: Materials Science and Engineering 940, 012106 (2020)

18. S. Barykin, O. Kalinina, I. Aleksandrov, E. Konnikov, V. Yadikin, and M. Draganov, Journal of Open Innovation: Technology, Market, and Complexity 6, 1 (2020)

19. S.Y. Barykin, I.V. Kapustina, S.M. Sergeev, and V.K. Yadykin, Journal of Open Innovation: Technology, Market, and Complexity 6, 1 (2020)

20. L. N. Borisoglebskaya, V. V. Provotorov, S. M. Sergeev, and E. S. Kosinov, IOP Conference Series: Materials Science and Engineering 537, (2019)

21. L.N. Borisoglebskaya, E.N. Provotorova, S.M. Sergeev, and A.P. Khudyakov, IOP Conference Series: Materials Science and Engineering 537 (2019)

22. L.N. Borisoglebskaya, E.N. Provotorova, and S.M. Sergeev, IOP Conference Series: Materials Science and Engineering 537 (2019)

23. S. Krasnov, S. Sergeev, A. Titov, and Y. Zotova, IOP Conference Series: Materials Science and Engineering 497 (2019)

24. S. Krasnov, S. Sergeev, E. Zotova, and N. Grashchenko, E3S Web of Conferences 110 (2019)

25. S. Krasnov, E. Zotova, S. Sergeev, A. Krasnov, and M. Draganov, IOP Conference Series: Materials Science and Engineering 618 (2019)

26. V. V. Provotorov, S. M. Sergeev, and A. A. Part, Vestnik Sankt-Peterburgskogo Universiteta, Prikladnaya Matematika, Informatika, Protsessy Upravleniya 15, 107 (2019) 
27. L.N. Borisoglebskaya, S.M. Sergeev, E.N. Provotorova, and A.A. Zaslavskiy, IOP Conference Series: Materials Science and Engineering 862 (2020)

28. O.V. Pilipenko, E.N. Provotorova, S.M. Sergeev, and O.V. Rodionov, Journal of Physics: Conference Series 1399 (2019)

29. A.A. Fedotov, S.M. Sergeev, E.N. Provotorova, T.V. Prozhogina, and O.Y. Zaslavskaya, IOP Conference Series: Materials Science and Engineering 862 (2020)

30. V.V. Provotorov, D.V. Danilevich, A.A. Fedotov, S.M. Sergeev, and O.J. Kravets, IOP Conference Series: Materials Science and Engineering 862 (2020)

31. L.N. Borisoglebskaya, E.N. Provotorova, and S.M. Sergeev, Journal of Physics: Conference Series 1399 (2019)

32. A.S. Golosnoy, V.V. Provotorov, S.M. Sergeev, L.B. Raikhelgauz, and O.J. Kravets, Journal of Physics: Conference Series 1399 (2019)

33. S.M. Sergeev, A.A. Kurochkina, O.V. Lukina, and V.E. Zasenko, IOP Conference Series: Materials Science and Engineering 918 (2020)

34. S. Sergeev and T. Kirillova, IOP Conference Series: Materials Science and Engineering 666 (2019)

35. V.V. Provotorov and E.N. Provotorova, Vestnik Sankt-Peterburgskogo Universiteta, Prikladnaya Matematika, Informatika, Protsessy Upravleniya 13, 209 (2017)

36. V.V. Provotorov, V.I. Ryazhskikh, and Y.A. Gnilitskaya, Vestnik SanktPeterburgskogo Universiteta, Prikladnaya Matematika, Informatika, Protsessy Upravleniya 13, 264 (2017)

37. A. S. Volkova and V.V Provotorov, Russian Mathematics 58, 1 (2014) 